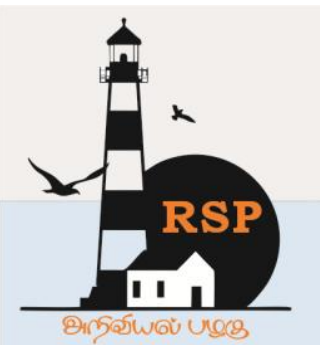

INTERNATIONAL RESEARCH JOURNAL ON ADVANCED SCIENCE HUB

-ISSN : $2582-437$
Open Access

RSP SCIENCE HUB

(The Hub of Research Ideas)

Available online at www.rspsciencehub.com

Special Issue of First International Conference on Information Technology, Computing \& Applications (ICITCA 2021)

\title{
EEG based Emotion Recognition and Classification: a Review
}

Ramprasad Kumawat ${ }^{1}$, Manish Jain ${ }^{2}$

${ }^{1}$ Ph.D Scholar, Department of Electrical and Electronics Engineering, Mandsaur Univesity, Mandsaur, Madhya Pradesh, India.

${ }^{2}$ Associate Professor, Department of Electrical and Electronics Engineering, Mandsaur Univesity, Mandsaur, Madhya Pradesh, India.

rp.kumawat@meu.edu.in ${ }^{l}$

\begin{abstract}
Emotion plays a vital role in medical research and interpersonal communication. Essentially feeling can be communicated verbally like discourse or non-verbally like outward appearance and physiological signals. A human emotion is complex physiological state which involves a physiological response, a person's experience and behavioral change. EEG measures electric current that are generated due to neuronal activities in the human brain. This paper provides an overview of comparative study of various techniques of emotion recognition from EEG signals. Our analysis is based on extracted features and classification methods of emotion recognition. We intended that, this study will be useful for newly researchers those entering in the field of emotion recognition.
\end{abstract}

Keywords: SVM, EEG, Emotion, Classifier, dataset and KNN

\section{Introduction}

Essentially feeling can be communicated verbally like discourse or non-verbally like outward appearance and physiological signals. Physiological signals are bio electrical signals that are control by the automatic nerves system $[1,2]$. The human computer interaction system (HCI) find lots of applications in biomedical engineering, neuromarketing, neuroscience and other areas of human life, which are affected by emotions. Because of increasing demand of HCI and automatic human emotion recognition. Currently emotional research focuses more in the diagnosis of depression, mental illness, and assists health care professionals to make accurate diagnosis [35]. Human emotion can be recognize through physiological signal like Electromyography (EMG), Electrocardiography(ECG), Gelvonic skin response and Electroencephalography(EEG) from all of these, EEG is more effective because it provide more accurate, non-invasive and convenient way of capturing brain signal. And also EEG is portable device [6,9]. Diagnosis of EEG signal varies with expertise. EEG recording require lot of time for manual inspection. And some time results may not be accurate because of artifact in the signals. So the processing an analysis of EEG signals can be done with the help of HCI technologies to get fast and accurate results. Using these technologies diagnosis of neuropsychiatric and neurological disorder such as Alzheimer, epilepsy and depressive disorder will be done [31]. Nowadays, researcher has shows extensive interest to make human emotion interaction with machine by proper brain computer interface [23].Literature survey covered recent methods used in physiological signal based emotion recognition; it will help for researcher working in this field. The remaining portion of this paper is organized as follows: Emotion is described in Section II. Characteristics and various rhythms of EEG 
www.rspsciencehub.com

signals is describes in section III. Section IV describes the various databases used and preprocessing of signals. Extracted features and various classification techniques have been describe in section Conclusion and future direction gives in section

\section{Emotion}

A human emotion is complex physiological state which involves a physiological response, a person's experience and behavioral change. An emotion is necessary component of human and significantly affects human's day by day activities including interaction, communication and learning etc.There are two perspectives towards representation of emotion. The first category indicate basic emotions through natural selection. There are eight basic emotions; fear, anger, disgust, sadness, curiosity, surprise, joy and acceptance. In the second category emotions are mapped into the Arousal, Valence and dominance[18]. Wast research on techniques of emotion recognition have been done over the past years and classified in to three catagories:

- Periphery physiological signals

- Facial expressions and voice

- Brain signal generated by central nervous system.

Some people cannot truly reflect their emotions into their facial expressions, because of some special reasons and a patient who suffers facial paralysis cannot express their filling. To solve these problems researchers had proposed emotion recognition methods based on Electrophysiological (EEG) signals and compared with other traditional methods [2].

\section{Electroencephalogram (EEG)}

The cortex is divided into the frontal, temporal, parietal, and occipital lobes(see fig.1)[18]. The frontal lobe is responsible for the conscious thought. The temporal lobe is responsible for the senses of smell and sound; The parietal lobe is responsible for integrating sensory information from various senses and the manipulation of objects and the at last, occipital lobe is responsible for the sense of sight. EEG estimates voltage fluctuations because of ionic current flows through neurons of the brain. A adult EEG signal, measured from the scalp is of range $10-100 \mu \mathrm{V}$. These signals are divided into specific ranges, namely the delta(1-4Hz), theta $(4-7 \mathrm{~Hz})$, alpha(8-
Volume 03 Issue 05S May 2021

$13 \mathrm{~Hz})$, beta $(13-30 \mathrm{~Hz})$ and gamma $(>30 \mathrm{~Hz})$ bands[18]. Delta waves are related with the unconscious brain, and occur during deep sleep. Theta waves are related with subconscious mind, and occur during sleeping and dreaming. Alpha waves are associated to relaxed mental state. Beta waves are an active state of mind. International 10/20 system (IS) (see fig.2)[18] are used for standard sets of locations of electrodes on the skull. The number 10 and 20 shows the distance between neibouring electrodes.

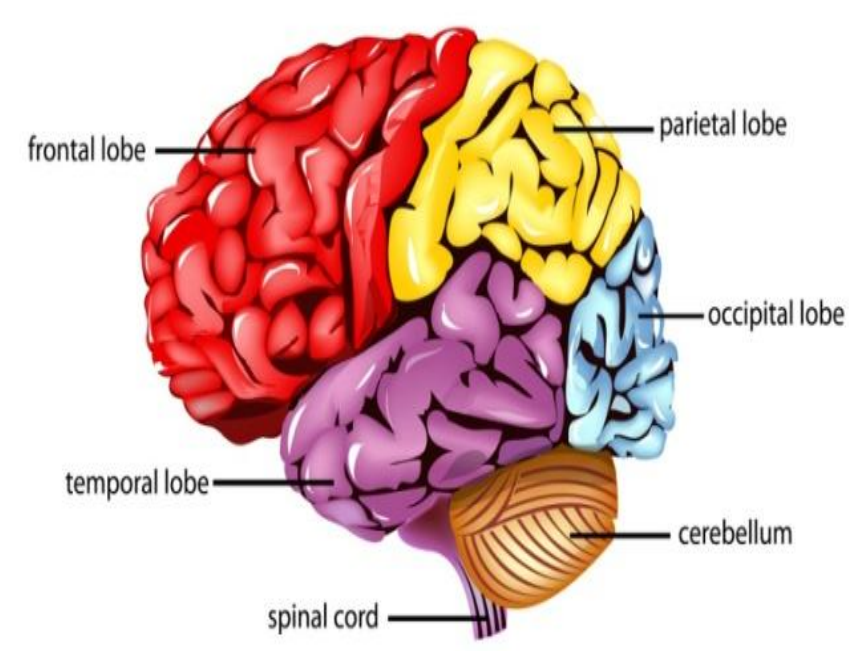

Fig. 1: Human brain subdivided in to temporal, frontal, occipital and parietal lobes. Adopted

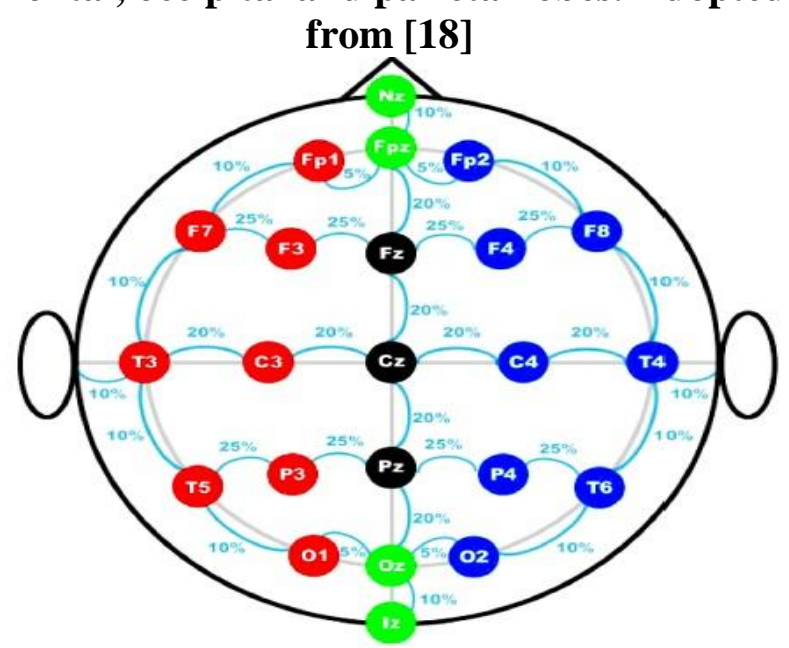

Fig. 2: International 10/20 system (IS)[18] The procedure of emotion recognition is as follows:

- User is exposed to the stimulus are tested.

- Changes in the voltage of human mind are recorded.

- From the EEG signals artifacts are removed. 
- After the removal of noise and artifact the signal is analyzed and the relevant features are extracted.
- Select appropriate classifier and train them from the training dataset and compute features and predict the signal.

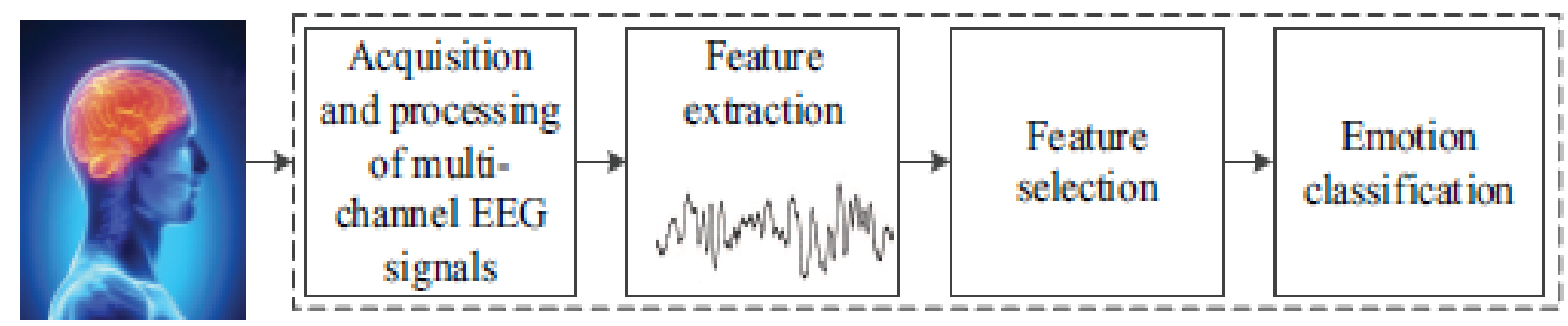

Fig.3: Procedure of EEG emotion recognition system

\section{Dataset and Data Preprocessing}

There are number of datasets are available publically that can be used for emotion recognition. Researchers mostly used DEAP, SEED and AMIGOS datasets.

a. DEAP[39] is widely used dataset in the EEG based emotion recognition. There are 32 subjects were participated in the Electrophysiological recording. They watch 40 one minute music videos. In the DEAP dataset total 32 channels were used to record.

b. SEED[40] 15 volunteers were participated in which each one was watching a small clips having distinct emotions. In SEED dataset total 62 channels were used for EEG recording.

c. AMIGOS[3] 40 volunteers were participated in which each one was watched a set of sixteen short videos. Each volunteers rated each video on the scale of two dimensional (valence and arousal).

Raw signal collected from EEG have noise and artifacts. Firstly we should remove this noise and artifacts from the raw signal. Artifacts removal is one of the most important step in emotion recognition. Artifacts are generated due to internal factors such as eye blinking, heart rate, muscles contraction and other environment factors like electrode position cable and recorders. There are many methods used for artifact removal, like linear regression, empirical mode decomposition, principal and component analysis etc.

\section{Feature Extraction and Classification}

Feature Extraction- For the better performance of biomedical (EEG) signals feature extractions are required. The main aim of feature extraction is to determine most important informative set of features to increase accuracy of the classifiers. A suitable feature extraction method converts one of many signals into a feature vector. In the machine learning algorithm researchers believe that suitable feature extraction is the key to make an efficient predictive model. There are several types of features based on frequency domain, Time domain and frequency time domain for the EEG signal diagnosis and analysis[23]. Feature ClassificationMachine learning is a subject of artificial intelligence. In the machine learning we train some network to build the predictive model. There are many supervised machine learning algorithm exist. There are various feature extraction methods are used by researchers to perform frequency domain, time domain and time frequency domain analysis of EEG signals. Fourier transform(FT), empirical mode decomposition(EMD), wavelet packet decomposition(WPD), wavelet transform, mRMR, etc are some of the feature extraction methods[31]. Extracted features are further classified using machine learning algorithms. Some of feature extraction techniques and classification techniques are discussed in this paper. In this section we have discuss machine learning techniques for emotion recognition. In[2] used an Ensemble Convolutional Neural Network (ECNN) model, which is used to automatically mine the correlation between multi-channel EEG signals and peripheral physiological signals to improve the emotion recognition accuracy. Author design five convolution networks and use global average pooling (GAP) layers instead of fully connected layers, and then the plurality voting strategy is adopted to establish these model. In[3] a deep belief-conditional random field (DBN-CRF) framework is used which is improved version of deep belief networks with glia chains (DBN-GC) and conditional random field. In this, feature vector sequence is extracted first from the EEG 
signals. Then, parallel DBN-GC models are utilized to obtain the feature sequence from EEG signals. And then conditional random field (CRF) model is used to generates the emotion state sequence. And at the last, K-nearest neighbor algorithm $(\mathrm{KNN})$ is used to estimate the emotion state. DEAP, SEED \& AMIGOS dataset is used in this. In [4] subject independent emotion recognition system using Discrete Wavelet Transform and MLP is used. This method is better than the earlier methods as it has used publically available dataset which contains data from 32 subjects from both the men and women. DEAP database is used and accuracy obtained is $58.50 \%$. In [6] a feature extraction technique based on double tree complex wavelet transform (DTCWT) and machine learning algorithm is used. In this a Neuroscan device is used for 16 subjects with video stimuli. Then band pass filter is used to remove artifects. And at the last, support vector machine (SVM) is used to classify emotions: calm, happy, and sad. And obtained the classification accuracy of $90.61 \%$. In [7] EMD method is utilized to decompose EEG signals with channels F3 and C4. A series of IMFs obtained by EMD are used to calculate SampEn values and to form feature vectors. These vectors are fed into SVM classifier for training and testing. The average accuracy of this method is $94.98 \%$ for DEAP dataset. In [11] a hierarchical bidirectional GRU model with attention mechanism (H-ATT-BGRU) is used for classification of emotion. The first layer of the model encodes the local correlation among the samples in an epoch, and the second layer encodes the temporal correlation among the EEG epochs in a sequence. The model uses attention mechanism at both sample and epoch levels. In [13] tunable-Q wavelet transform (TQWT) is used for the classification of various emotions of EEG signals. tunable-Q wavelet transform divides EEG signal into subbands and then time-domain features are extracted from these subbands. These features are used as an input to machine learning classifier for the classification of happy, fear, sad, and relax emotions. In [14] phase locking value (PLV) graph convolutional neural networks [PGCNN] is used which is the improved model of PLV and GCNN. The PLV has the ability to segregate phase and amplitude component in the
EEG signal and determine inter channel correlation information. In [16] a hybrid feature extraction method is used in Empirical Mode Decomposition (EMD) domain with combination of Sequence Backward Selection (SBS) for EEG emotion recognition. In this detail information of multi scale components of EEG signal was extracted and optimal features was selected for emotion classification. Model is tested on DEAP dataset, in which the Valance and Arousal dimension emotional states are classified by $\mathrm{K}$ nearest neighbor (KNN) and support vector machine (SVM). In[19] a feature extraction technique Teager-Kaiser Energy Operator (TKEO) is used along with k-nearest neighbor (KNN), neural network (NN) and Classification Tree (CT) classifiers for the emotion recognition from EEG signal. This study determines the performance and accuracy of emotion recognition which is further used for stress identification. In [20] dual tree complex wavelet transform (DT-CWT) was used to decomposed the EEG into five different sub bands from which different features were extracted using frequency, time and non linear analysis. In this deep simple recurrent units (SRU) network is adopted which is not only able to processing a sequence data but also has the ability to resolve the problem of long term dependencies occurs in normal recurrent neural network(RNN). Positive neutral and negative emotions were identified from EEG signals from emotion recognition system. In [23] a hybrid method of principal component analysis (PCA) and t-statistics is used for feature extraction. This spatial PCA was implemented to reduce signal dimensionality and select features based on the t-statistical. In [27] a GPSO algorithm for tuning the hyperparameters in the CNN model is used, and applies the optimized CNN model to the EEG emotion recognition. In [29] mRMR feature selection method is used after the preprocessing step of EEG signal to improve the accuracy of SVM emotion classifier on twodimension(Valence and Arousal) emotions model. In [30] Liquid State Machines (LSM) algorithm is method of feature extraction and used to recognize the emotion state of an individual from EEG dataset. In this author identified arousal, valence and liking emotional states from the available dataset.[35-41]. 
Table: 1. Various Algorithms Used in Emotional State Recognition

\begin{tabular}{|c|c|c|c|c|c|c|}
\hline $\begin{array}{l}\text { Refer } \\
\text { ence }\end{array}$ & Dataset & $\begin{array}{l}\text { Features } \\
\text { extracted }\end{array}$ & Classifiers & $\begin{array}{l}\text { No. of } \\
\text { channel }\end{array}$ & Emotional State & Accuracy \\
\hline$[1]$ & $\begin{array}{l}\text { DEAP } \\
\& \\
\text { TYUT } \\
2.0\end{array}$ & $\begin{array}{c}\text { Hajorth } \\
\text { Parameters, } \\
\text { FD, DE,WE } \\
\text { and FC }\end{array}$ & $\begin{array}{c}\text { SVM with } \\
\text { Gaussian Kernel }\end{array}$ & $\begin{array}{l}32 \text { and } \\
64\end{array}$ & $\begin{array}{c}\text { Sadness, Anger, } \\
\text { happiness, } \\
\text { surprise and } \\
\text { neutral }\end{array}$ & $\begin{array}{c}\text { DEAP- } \\
84.67 \% \\
\text { TYUT2.0- } \\
89.64 \%\end{array}$ \\
\hline [2] & DEAP & $\begin{array}{c}\text { Root mean } \\
\text { square, } \\
\text { Stochastic } \\
\text { gradient } \\
\text { descent(SGD) }\end{array}$ & ECNN & 32 & $\begin{array}{c}\text { Arousal, Valence, } \\
\text { liking and } \\
\text { Dominance }\end{array}$ & $82.92 \%$ \\
\hline [3] & $\begin{array}{l}\text { DEAP, } \\
\text { SEED \& } \\
\text { AMIGO } \\
\text { S }\end{array}$ & $\begin{array}{c}\text { Mean, } \\
\text { Variance, Zero } \\
\text { crossing rate, } \\
\text { approximate } \\
\text { entropy and } \\
\text { PSD } \\
\end{array}$ & $\begin{array}{c}\text { Deep belief } \\
\text { conditional } \\
\text { random field } \\
\text { (DBN-CRF), } \\
\text { DBN-GC and } \\
\text { KNN } \\
\end{array}$ & $32 \& 62$ & $\begin{array}{l}\text { Arousal and } \\
\text { Valence }\end{array}$ & $\begin{array}{l}\text { Arousal- } \\
76.13 \%, \\
\text { Valence- } \\
77.02 \%\end{array}$ \\
\hline [4] & DEAP & $\begin{array}{c}\text { Wavelet } \\
\text { features (WF) }\end{array}$ & $\begin{array}{c}\text { Multilayer } \\
\text { Perceptron neural } \\
\text { network (MLP) }\end{array}$ & 1 & Happy and Sad & $58.50 \%$ \\
\hline [6] & DEAP & $\begin{array}{c}\text { Wavelet } \\
\text { features (WF) }\end{array}$ & $\begin{array}{c}\text { Deep Neural } \\
\text { network (DNN) }\end{array}$ & 2 & $\begin{array}{l}\text { Valence and } \\
\text { Arousal }\end{array}$ & $\begin{array}{l}\text { Valenc- } \\
62.5 \% \text { \& } \\
\text { Arousal- } \\
64.25 \%\end{array}$ \\
\hline [8] & DEAP & $\begin{array}{l}\text { NMI based } \\
\text { channel } \\
\text { selection }\end{array}$ & SVM & 32 & $\begin{array}{l}\text { Valence and } \\
\text { Arousal }\end{array}$ & $\begin{array}{c}\text { Arousal- } \\
73.64 \%, \text { Vale } \\
\text { nce } 74.4 \%\end{array}$ \\
\hline [9] & DEAP & $\begin{array}{l}\text { Statistical } \\
\text { features and } \\
\text { frequency } \\
\text { domain } \\
\text { features }\end{array}$ & $\begin{array}{c}\text { Sparse } \\
\text { Discriminative } \\
\text { Ensemble } \\
\text { Learning (SDEL) } \\
\text { with DNN and } \\
\text { CNN }\end{array}$ & 32 & $\begin{array}{l}\text { HAHV,LALV } \\
\text {,HALV, LAHV }\end{array}$ & $\begin{array}{l}\text { Arousal- } \\
70.1 \% \text { and } \\
\text { Valence- } \\
77.4 \%\end{array}$ \\
\hline$[10]$ & NA & $\begin{array}{c}\text { Hjorth } \\
\text { parameters(acti } \\
\text { vity, mobility, } \\
\text { and } \\
\text { complexity) }\end{array}$ & $\begin{array}{c}\text { deep- } \\
\text { learning(bagging, } \\
\text { boosting, staking } \\
\text { and voting), } \\
\text { naive-Bayes, } \\
\text { LDA, KNN, } \\
\text { SVM, }\end{array}$ & 14 & $\begin{array}{l}\text { happy, calm, sad, } \\
\text { and scared) }\end{array}$ & $76.60 \%$ \\
\hline$[11]$ & DEAP & $\begin{array}{c}\text { PSD, SFFS and } \\
\text { AR }\end{array}$ & $\begin{array}{c}\text { CNN, LSTM, } \\
\text { hierarchical } \\
\text { bidirectional } \\
\text { Gated Recurrent } \\
\text { Unit (GRU) model } \\
\text { (H-ATT-BGRU) }\end{array}$ & $32 \& 64$ & $\begin{array}{l}\text { Arousal and } \\
\text { Valence }\end{array}$ & $\begin{array}{l}\text { Valence- } \\
69.9 \% \text { and } \\
\text { Arousal- } \\
65.5 \%\end{array}$ \\
\hline$[12]$ & $\begin{array}{c}\text { DEAP } \\
\text { and }\end{array}$ & $\begin{array}{c}\text { Standard } \\
\text { Deviation, first } \\
\end{array}$ & $\begin{array}{l}\text { KNN, Decision } \\
\text { tree \& Random }\end{array}$ & $32 \& 64$ & $\begin{array}{c}\text { Negative, Calm } \\
\text { and Positive }\end{array}$ & $\begin{array}{c}\text { DEAP- } \\
63.09 \% \text { and }\end{array}$ \\
\hline
\end{tabular}




\begin{tabular}{|c|c|c|c|c|c|c|}
\hline & SEED & $\begin{array}{l}\text { and second } \\
\text { order } \\
\text { differential } \\
\text { features }\end{array}$ & Forest & & & SEED-75\% \\
\hline [14] & $\begin{array}{l}\text { DEAP } \\
\text { and } \\
\text { SEED }\end{array}$ & $\begin{array}{c}\text { PSD, } \\
\text { Differential } \\
\text { Asymmetry(D } \\
\text { ASM), } \\
\text { Differential } \\
\text { Entropy(DE), } \\
\text { Rational } \\
\text { Asymmetry(R } \\
\text { ASM) and } \\
\text { Differential } \\
\text { Caudality(DCA } \\
\text { U) }\end{array}$ & $\begin{array}{l}\text { phase-locking } \\
\text { value (PLV) graph } \\
\text { convolutional } \\
\text { neural networks } \\
\text { (P-GCNN) }\end{array}$ & $32 \& 64$ & $\begin{array}{c}\text { valence, arousal, } \\
\text { and dominance } \\
\text { using }\end{array}$ & $\begin{array}{c}\text { Valence- } \\
73.31 \% \text {, } \\
\text { Arousal- } \\
77.03 \text { and } \\
\text { Dominance- } \\
79.20 \%\end{array}$ \\
\hline [16] & DEAP & $\begin{array}{c}\text { spectrum } \\
\text { centroid } \\
\text { and Lempel- } \\
\text { Ziv } \\
\text { Complexity } \\
\text { (LZC) }\end{array}$ & $\mathrm{KNN}$ and $\mathrm{SVM}$ & 32 & Valence, Arousal & $\begin{array}{l}\text { Valence- } \\
86.46 \% \\
\text { Arousal- } \\
84.90 \%\end{array}$ \\
\hline [19] & DEAP & $\begin{array}{c}\text { Energy, } \\
\text { Feature } \\
\text { vector(FV) }\end{array}$ & $\begin{array}{c}\text { k-nearest neighbor } \\
(\mathrm{KNN}), \text { neural } \\
\text { network(NN) and } \\
\text { Classification } \\
\text { Tree (CT) }\end{array}$ & 2 & $\begin{array}{l}\text { Positive and } \\
\text { Negative }\end{array}$ & $83.33 \%$ \\
\hline [20] & SEED & $\begin{array}{c}\text { MAV, PSD, } \\
\text { fractal } \\
\text { Dimension(FD) } \\
\text {, DE } \\
\end{array}$ & $\begin{array}{l}\text { Deep Simple } \\
\text { Recurrent } \\
\text { Unit(SRU) }\end{array}$ & 64 & $\begin{array}{c}\text { Positive, Negative } \\
\text { and Neutral }\end{array}$ & NA \\
\hline [23] & SEED & $\begin{array}{c}\text { Standard } \\
\text { Deviation(SD), } \\
\text { Mean Absolute } \\
\text { deviation(MA } \\
\text { D), Median } \\
\text { Absolute } \\
\text { Deviation(Med } \\
\text { AD), FD, PSD } \\
\text { and Spectral } \\
\text { Energy } \\
\end{array}$ & $\begin{array}{c}\mathrm{ANN}, \mathrm{SVM}, \mathrm{LDA} \\
\text { and KNN }\end{array}$ & 64 & $\begin{array}{c}\text { Positive, Negative } \\
\text { and Neutral }\end{array}$ & $\begin{array}{c}\text { ANN- } \\
86.57 \% \\
\text { SVM- } \\
85.85 \% \\
\text { LDA- } \\
82.50 \% \\
\text { KNN- } \\
73.42 \%\end{array}$ \\
\hline [24] & $\begin{array}{l}\text { Own } \\
\text { data }\end{array}$ & $\begin{array}{c}\text { sample } \\
\text { entropy, Tsallis } \\
\text { entropy, } \\
\text { Higuchi fractal } \\
\text { dimension, and } \\
\text { Hurst exponent }\end{array}$ & $\begin{array}{l}\text { multi-class least } \\
\text { squares support } \\
\text { vector machine } \\
\text { (MC-LS-SVM) }\end{array}$ & NA & $\begin{array}{l}\text { happy, fear, sad, } \\
\text { and relax }\end{array}$ & $\begin{array}{c}\text { happy- } \\
92.79 \%, \\
\text { fear }=87.62 \% \\
\text { sad- } \\
88.98 \% \text {, and } \\
\text { relax- } \\
93.13 \%\end{array}$ \\
\hline [25] & DEAP & PSD & $\begin{array}{c}\text { Deep Neural } \\
\text { network [DNN] }\end{array}$ & 4 & $\begin{array}{l}\text { Arousal and } \\
\text { Valence }\end{array}$ & \\
\hline
\end{tabular}




\begin{tabular}{|c|c|c|c|c|c|c|}
\hline [26] & DEAP & $\begin{array}{c}\text { Statistical } \\
\text { features: } \\
\text { power, mean, } \\
\text { standard } \\
\text { deviations, } \\
\text { narmalized } \\
\text { difference, } \\
\text { Hjroth } \\
\text { parameters: } \\
\text { mobility, } \\
\text { complexity. } \\
\text { Fractal } \\
\text { Dimention(FD) }\end{array}$ & $\begin{array}{l}\text { Hypergraph } \\
\text { Partitioning }\end{array}$ & 32 & $\begin{array}{c}\text { arousal, Valence, } \\
\text { Dominance and } \\
\text { Liking }\end{array}$ & $\begin{array}{c}\text { Arousal- } \\
59.77 \% \\
\text { Valence- } \\
51.88 \% \\
\text { Liking- } \\
62.11 \% \\
\text { Dominance- } \\
63.75 \%\end{array}$ \\
\hline [28] & SEED & $\begin{array}{l}\text { Dynamic } \\
\text { Sample } \\
\text { Entropy }\end{array}$ & SVM & 62 & $\begin{array}{l}\text { Positive and } \\
\text { Negative }\end{array}$ & $85.11 \%$ \\
\hline [29] & DEAP & $\begin{array}{c}\text { Hjorth } \\
\text { parameters, } \\
\text { Statistical } \\
\text { features and } \\
\text { Fractal } \\
\text { Dimension }\end{array}$ & SVM & 32 & $\begin{array}{l}\text { Arousal and } \\
\text { Valence }\end{array}$ & $\begin{array}{l}\text { Aroual- } \\
60.7 \% \\
\text { Valence- } \\
62.33 \%\end{array}$ \\
\hline [30] & DEAP & NA & $\begin{array}{c}\text { Decision } \\
\text { Tree(DT) and } \\
\text { Linear } \\
\text { Discriminate } \\
\text { Analysis(LDA) }\end{array}$ & 32 & $\begin{array}{l}\text { Arousal, Valence } \\
\text { and liking }\end{array}$ & NA \\
\hline [32] & $\begin{array}{c}\text { Own } \\
\text { dataset }\end{array}$ & $\begin{array}{c}\text { Fractal } \\
\text { Dimension } \\
\end{array}$ & SVM & 14 & $\begin{array}{c}\text { Calm, anger and } \\
\text { Happiness }\end{array}$ & $60 \%$ \\
\hline [33] & DEAP & $\begin{array}{l}\text { Mean and } \\
\text { standard } \\
\text { Devaition }\end{array}$ & $\begin{array}{c}\text { probabilistic } \\
\text { neural network } \\
(\mathrm{PNN})\end{array}$ & & $\begin{array}{l}\text { HAHV,LALV } \\
\text { „HALV, LAHV }\end{array}$ & $82.01 \%$ \\
\hline [34] & DEAP & $\begin{array}{c}\text { (standard } \\
\text { deviation, } \\
\text { mean, kurtosis } \\
\text { and skewness) }\end{array}$ & $\begin{array}{c}\text { Linear } \\
\text { Discriminate } \\
\text { Analysis(LDA) }\end{array}$ & 13 & $\begin{array}{c}\text { Positive, } \\
\text { Negative, Angry } \\
\text { and Harmony }\end{array}$ & $82 \%$ \\
\hline
\end{tabular}

\section{Conclusion and Future Direction}

In the analysis of brain signal we use EEG signals, as it is non-invasiveness high temporal resolution and safe nature. In this study we have surveyed the various methods of emotion recognition from EEG signals. A comparative analysis of EEG signals have been done in which feature extraction and classification methods are discussed. Our main focus on classification techniques and extracted features. From the analysis, we concluded that each and every stage has its own role in EEG signals analysis. Each and every state play a very important role in pre-processing EEG signals and developing novel model. In pre-processing unwanted signals, noise and artifacts are removed from the raw EEG signals. In second state, feature extraction algorithms are used to represent high dimensional signal into discrete features. Then, if data suffers from overfitting problem, feature reduction techniques can be used to reduce the computational cost. And at last signals are classified with different machine learning algorithms and deep learning algorithms for emotion recognition. From the study of various research papers it is concluded that accuracy of deep learning algorithm is better than machine 


\section{www.rspsciencehub.com}

learning algorithms. In future, we will made efforts in more advanced algorithms for feature extractions and classification techniques from the publically available datasets.

\section{References}

[1].Guijun Chen, Xueying Zhang, Ying Sun and Jing Zhang, Emotion feature analysis and recognition based on reconstructed EEG

[2]. sources. IEEE ACCESS, 2017, p. 1-11.

[3].Haiping Huang, Zhenchao Hu, Wenming Wang, and Min $\mathrm{Wu}$, Multimodal Emotion Recognition Based on Ensemble Convolutional Neural Network. IEEE ACCESS, 2020; 8, pp. 3265-71.

[4].Hao Chao And Yongli Liu, Emotion Recognition From Multi-Channel EEG Signals by Exploiting the Deep Belief-Conditional Random Field Framework. IEEE ACCESS, 2020; 8, pp. 32002-12.

[5].Pallavi Pandey and K. R. Seeja, Emotional State Recognition with EEG Signals Using Subject Independent Approach. Springer Nature Singapore, 2019, p. 117-24.

[6]. Xin Xu1, Yiwei Zhang, Minghong Tang, Hong Gu, Shancheng Yan, and Jie Yang, Emotion Recognition Based on Double Tree Complex Wavelet Transform and Machine Learning in Internet of Things. IEEE ACCESS, 2017, p. 1-9.

[7].Pallavi Pandey and K. R. Seeja, This paper proposes a subject-independent emotion recognition system using DWT and deep neural network. International Conference on Innovative Computing and Communications Proceedings of ICICC-2018, p. 41-46.

[8]. Yong Zhanga, Xiaomin Ji a, Suhua Zhang, An approach to EEG-based emotion recognition using combined feature extraction method. Elsevier, 2016, p. 152-57.

[9].Zhong-Min Wang, Shu-Yuan $\mathrm{Hu}$, and Hui Song, Channel Selection Method for EEG Emotion Recognition Using Normalized Mutual Information.IEEE ACCESS, 2019;7, P. 143303-13.

[10].Habib Ullah, Muhammad Uzair, Arif Mahmood, Mohib Ullah, Sultan Daud Khan, Faouzi Alaya Cheikh, Internal Emotion Classification Using EEG Signal with Sparse
Volume 03 Issue 05S May 2021

Discriminative Ensemble. IEEE ACCESS, 2018; 4, p. 1-11.

[11].Raja Majid Mehmood 1, Ruoyu Du 2 and Hyo Jong Lee, Optimal feature selection and Deep Learning Ensembles Method for emotion recognition from human brain EEG sensors. IEEE ACCESS, 2017.

[12].J. X. Chen, D. M. Jiang, and Y. N. Zhang, A Hierarchical Bidirectional GRU Model With Attention for EEG-Based Emotion Classification. IEEE, 2019; 7, p.118530-40.

[13]. Chunmei Qing, Rui Qiao, Xiangmin Xu and Yongqiang Cheng, Interpretable Emotion Recognition Using EEG Signals. IEEE,2019;20, p.1-10.

[14].Anala Hari Krishna, Aravapalli Bhavya Sri, Kurakula Yuva Venkata Sai Priyanka, Sachin Taran1, Varun Baja, Emotion classification using EEG signals based on tunable- $Q$ wavelet transform. IET Science, Measurement \& Technology, 2019;13(3), p. 375-380.

[15].Zhongmin Wang, Yue Tong, and Xia Heng, Phase-Locking Value Based Graph Convolutional Neural Networks for Emotion Recognition. IEEE, 2019;7, p. 93711-22.

[16].Houtan Jebelli, Mohammad Mahdi Khalili, and SangHyun Lee, A Continuously Updated, Computationally Efficient Stress Recognition

Framework Using Electroencephalogram (EEG) by Applying Online Multi- task Learning Algorithms (OMTL) IEEE Journal of Biomedical and Health Informatics, 2018, p.112.

[17]. Zhen-Tao Liu, Member, IEEE, Qiao Xie, Min Wu, Senior Member, IEEE, Wei-Hua Cao, Dan-Yun Li, and Si-Han Li, Electroencephalogram Emotion Recognition Based on Empirical Mode Decomposition and Optimal Feature Selection. IEEE Transactions on Cognitive and Developmental Systems, 2018, p. 1-10.

[18].Zirui Lan, Olga Sourina, Lipo Wang, Reinhold Scherer, Gernot R. Müller-Putz, Domain Adaptation Techniques for EEG-based Emotion Recognition: A Comparative Study on Two Public Datasets. IEEE Transactions on Cognitive and Developmental Systems,2018, p. $1-10$. 
[19].Soraia M. Alarc ao, and Manuel J. Fonseca, Emotions Recognition Using EEG Signals: A Survey. IEEE Transactions on Affective Computing, 2017, p. 1-20.

[20].Prashant Lahane, Mythili Thirugnanam, Human Emotion Detection and Stress Analysis using EEG Signal. International Journal of Innovative Technology and Exploring Engineering, 2019;8, p. 96-100.

[21].Chen Wei, Lan-lan Chen*, Zhen-zhen Song, Xiao-guang Lou, Dong-dong Li, EEG-based emotion recognition using simple recurrent units networkand ensemble learning. Biomedical Signal Processing and Control, Elsevier, 2019; 58, p.1-13.

[22].Jingxia Chen, Dongmei Jiang, Yanning Zhang, Pengwei Zhang, Emotion recognition from spatiotemporal EEG representations with hybrid convolutional recurrent neural networks via wearable multi-channel headset. Computer Communications, Elsevier, 2020;154, p. 58-65.

[23].Prashant Lahane1, Arun Kumar Sangaiah2, An Approach to EEG Based Emotion Recognition and Classification using Kernel Density Estimation. International Conference on Intelligent Computing, Communication \& Convergence (ICCC-2014), Procedia Computer Science, Elsevier, 2015, p.574-81.

[24].Md. Asadur Rahman, Md. Foisal Hossain, Mazhar Hossain, Rasel Ahmmed, Employing PCA and t-statistical approach for feature extraction and classification of emotion from multichannel EEG signal. Egyptian Informatics Journal, Science Direct, 2019; 21, p. 23-35.

[25]. Sachin Taran, Varun Bajaj, Emotion recognition from single-channel EEG signals using a two-stage correlation and instantaneous frequency-based filtering method. Computer Methods and Programs in Biomedicine, Elsevier, 2019;173, p.157-165.

[26].Pallavi Pandey, K.R. Seeja, Subject Independent Emotion recognition from EEG using VMD and DeepLearning. Journal of King Saud University - Computer and Information Sciences, 2019, 2019, p.1-18.

[27].Zhen Liang, Shigeyuki Oba, Shin Ishii, An unsupervised EEG decoding system for human emotion recognition. Neural Networks, Elsevier, 2019, 116, p. 157-168.
[28].Zhongke Gao, Yanli Li, Yuxuan Yang, Xinmin Wang, Na Dong, Hsiao-Dong Chiang, A GPSO-optimized Convolutional Neural Networks for EEG-based Emotion Recognition. Neurocomputing, 2019, p. 1-14.

[29].Yun Lu, Mingjiang Wang, Wanqing Wuc, Yufei Han, Qiquan Zhang, Shixiong Chen, Dynamic entropy-based pattern learning to identify emotions from EEG signals across individuals. Measurement, Elsevier, 2019;150, 107003-14.

[30].John Atkinsona, Daniel Campos, Improving BCI based emotion recognition by combining EEG features election and kernel classifiers. Expert Systems With Applications, Elsevier, 2016; 47, p. 35-41.

[31].Obada Al Zoubia, Mariette Awada, Nikola K. Kasabov, Anytime multipurpose emotion recognition from EEG data using a Liquid State Machine based framework. Artificial Intelligence in Medicine, Elsevier, 2018, p. 18.

[32].Ashima Khosla, Padmavati Khandnor, Trilok Chand, A comparative analysis of signal processing and classification methods for different applications based on EEG signals. Biocybern Biomed Eng, Elsevier, 2020, p. 142.

[33].Barjinder Kaur, , Dinesh Singh, Partha Pratim Roy, EEG Based Emotion Classification Mechanism in BCI. International Conference on Computational Intelligence and Data Science (ICCIDS 2018), Procedia Computer Science, Elsevier, 2018;132, p. 752-758.

[34].Rahul Sharmaa, Ram Bilas Pachorib, Pradip Sircar, Automated emotion recognition based on higher order statistics and deep learning algorithm. Biomedical Signal Processing and Control, Elsevier, 2020; 58, P. 101867-76.

[35].Debashis Das Chakladara, Sanjay Chakraborty, EEG based emotion classification using "Correlation Based Subset Selection. Biologically Inspired Cognitive Architectures, Elsevier, 2018.

[36].Mitul Kumar Ahirwal, Mangesh Ramaji Kose, Emotion Recognition System based on EEGsignal: A Comparative Study of Different Features and Classifiers. International Conference on Computing Methodologies and 
Communication (ICCMC 2018), IEEE Explore.

[37].Thejaswini S, K M Ravikumar, Jhenkar L, Aditya Natraj, Abhay K K, Analysis of EEG Based EmotionDetection of DEAP and SEEDIV Databases using SVM. International Journal of Recent Technology and Engineering (IJRTE), 2019.

[38].Moon Inder Singh, Mandeep Singh, Development of a real time emotion classifier based on evoked EEG. Biocybernetics and Biomedical Engineering, Elsevier, 2017; 37, p. 498-509.

[39].Nitin Kumar, Kaushikee Khaund, Shyamanta M. Hazarik, Bispectral Analysis of EEG for EmotionRecognition. 7th International conference on Intelligent Human Computer Interaction, Procedia Computer Science, 2016; 84, p. 31-35.

[40].http://www.eecs.qmul.ac.uk/mmv/datasets/de ap/

[41].http:/www.bcmi.sjtu.edu.cn/ seed/download. html/ 